\title{
A botanical nano ozonated erotic oil composition with maximum bioavailability for men
}

\begin{abstract}
The present patent-pending invention relates to a topical pharmaceutical nano ozonated composition(with maximum bioavailability) for male sexual response, including male sexual dysfunction, such as male sexual arousal, vitality, erection disorders, premature ejaculation, and sexual pain disorders, and enhancing male sexual pleasure and satisfaction of the male sexual experience as well as penis enlargement. The composition for treating this problem preferably formed of a natural oil mix, fenugreek oil, watermelon oil, ginger oil and ozonated olive oil. In particular the composition is applied on penis and testicles to enhance pleasure and delay ejaculation for men during intercourse. Observed results showed excellent results for all ages of men with no adverse or side effects.
\end{abstract}

Volume 5 Issue I - 2018

\author{
Awad Mansour,' Ammar Mansour² \\ 'University of Akron, USA \\ ${ }^{2}$ Essraa Hospital, Jordan
}

Correspondence: Awad Mansour, University of Akron, $\mathrm{OH}$, USA, Tel 9622-7278278, Email profmansour@gmail.com

Received: November 25, 2017 | Published: February 07, 2018
Abbreviations: ED, erection dysfunction; PE, premature ejaculation; ASN, applied science and nutrition

\section{Description of the invention}

Erection dysfunction is a condition that occurs when someone has difficulty reaching erection or suffers from premature ejaculation during intercourse process, even when they're sexually aroused and there's sufficient sexual stimulation. When this condition occurs in men, it's known as male erection dysfunction (ED). Erection and premature ejaculation are intense feelings of release during sexual stimulation. They can vary in intensity, duration, and frequency from one man to another. Many men have difficulty reaching hard erection and long-time ejaculation to satisfy a partner even after ample sexual stimulation. In fact, erection dysfunction affects approximately one in three men because of oil age, diabetes, high blood pressure or heart or kidney disorders. Premature ejaculation is a common problem from which more than $50 \%$ of men suffer.

Huge number of studies focused on male erectile dysfunction (ED) and Premature Ejaculation (PE) progressed rapidly in the past twenty years and led to new therapeutic chemical drugs and formulations, hundreds of millions of ED sufferers are still waiting alternative natural safe products to solve this essential problem in men and women lives. Accordingly the present invention relates to all aspects of managing the male sexual response, including male erection dysfunction (ED), and premature ejaculation (PE). In particular the present invention relates to compositions, articles of manufacture, methods of preparation thereof, methods of use thereof, etc., for conditions, disorders, and diseases related to male reproductive physiology systems, especially those involved in the male sexual response.

Compositions comprising botanical extracts, active agents, etc., can be produced and used in accordance with the present invention that is useful to treat or affect the male sexual response. For example, the present invention relates to compositions, preferably for topical or local use, which comprises one or more of the following ingredients, including, but not limited to, fenugreek oil, watermelon oil and ozonated olive oil. The compositions can produce one or more of the following pharmacological effects, including, but not limited to, increases in localized nitric oxide, anti-oxidation, vasodilation, smooth muscle relaxation, etc. Australian researchers at the University of Queensland ${ }^{1}$ working with colleagues at Applied Science and Nutrition (ASN), a Brisbane-based company that specializes in scientific and consulting services to the nutraceutical and complementary medicine fields, found a natural, herbal way to increase libido and treat ED. In a double-blind, randomized, placebo-controlled study, the scientists found that daily supplements containing an extract from fenugreek appeared to enhance male libido and normalize testosterone levels. Fenugreek (known by its botanical name Trigonella foenum-graecum), is a plant used both as an herb (the leaves) and a spice (the seed); it's a common ingredient in many curries. For the new study, which was just published in the journal Phytotherapy Research, the research team used a supplement containing a standardized extract of fenugreek.

The Australian scientists recruited 60 healthy men between the ages of 25 and 52 for the study. The research participants were randomly assigned to receive $600 \mathrm{mg}$ of Testofen daily or an oral placebo. After six weeks, the majority of men receiving the fenugreek extract reported improvements in libido and quality of sexual performance. Overall, $66 \%$ of the men receiving the herb extract reported improvements in the time it took to have another erection after intercourse, $82 \%$ said they had more energy in general and 56\% said they experienced an improved sense of well-being after taking the supplement for a little over a month. Several studies of Universities as Almeria University in Spain, ${ }^{2}$ says that Olive Oil and even tomato can help to prevent and improve erection, lycopene from tomatoes with Olive Oil which help that to be absorbed for our body make the perfect combination.

According to the institute's director, urologist Dr. Juan Carlos Ruiz de la Roja, ${ }^{3}$ the scientific explanation is very simple. "Lycopene and olive oil has been shown to have a vasodilatory effect due to the liberation of nitric oxide, which improves blood flow overall, including to the penis, thus improving erection". The top benefits of ozonated essential oils ${ }^{4}$ ozonated oil holds a variety of health benefits, but is most commonly used for conditions of the skin and external body, much as you would use a therapeutic salve or cream. As a massage paste, the ozonide acts as an antioxidant, allowing lactic acid and toxins to be released from the pores of the skin. Other benefits and uses include: 
a. Stimulation of growth for skin cells.

b. Speeds up healing-time for skin wounds and ulcers.

c. Cleans and sterilizes the epidermis.

d. Reduces swelling and redness.

e. Calms the nerves and reduces the amount of pain in skin conditions.

f. May increase cellular function and cellular memory.

g. Acts as a free radical scavenger on harmful toxins.

h. Acts as a moisturizing facial conditioner (leave on for $20 \mathrm{minu}-$ tes and promptly remove).

i. Aids in healing processes and reduces scarring.

j. Promotes healthy skin conditions.

k. Good for removing makeup.

1. Good for animal wounds, as it is all natural, and can be licked $\mathrm{m}$. Sunburn of the skin.

$\mathrm{m}$. Insect bites and stings.

n. Infections of the sebaceous glands (sweat).

o. Can be used as a natural under arm deodorant.

p. Burns - One of the best natural remedies.

q. Sore muscles (rub as a massage paste).

r. Cellulite Reduction.

s. Wrinkles u. Ringworm.

t. Bed Sores.

u. Cuts \& Burns.

v. Skin Yeast.

w. Carbuncles.

x. Diaper Rash.

y. Tinea Versicolor.

z. Dermatitis.

According to a researcher in $2008,{ }^{5}$ watermelon may be a natural Viagra. That's because the popular summer fruit is richer than experts believed in an amino acid called citrulline, which relaxes and dilates blood vessels much like Viagra and other drugs meant to treat erectile dysfunction (ED). According to Dr. Bhimu Patil, director of Texas A $\&$ M's Fruit and Vegetable Improvement Center in College Station, when watermelon is consumed, citrulline is converted to arginine through certain enzymes. Arginine is an amino acid that works wonders on the heart and circulation system and maintains a good immune system, Patil said. "The citrulline-arginine relationship helps heart health, the immune system and may prove to be very helpful for those who suffer from obesity and type 2 diabetes," said Patil. "Arginine boosts nitric oxide, which relaxes blood vessels, the same basic effect that Viagra has, to treat erectile dysfunction and maybe even prevent it." How much watermelon does it take to treat erectile dysfunction (ED). The researcher doesn't have an answer for that. He does know that a typical 4-ounce serving of watermelon (about 10 watermelon balls) has about 150 milligrams of citrulline. But he can't say how much citrulline is needed to have Viagra-like effects.

Extensive research on treating impotence and Erectile Dysfunction ${ }^{6}$ has not only led to newer and effective medicines but also it has led to the discovery of the importance of aphrodisiac foods in men sex life. Throughout history, many foods, drinks, and behaviors have had a reputation for making sex more attainable and pleasurable. Kathi Keville and Peter Corn, authors of "Herbs for Health and Healing," stated that poor circulation may cause impotence in men because damaged or impaired blood vessels simply fail to fill with blood and expand which is required to attain a strong erection. They also say that certain herb, such as ginger, helps to increase dilation in blood vessels and improve circulation. Ginger have been used since ages as a herb in preparing food as well as used as an alternative to cure common ailments. According to herbal books, it has been stated that the active compounds in ginger are gingerol, shogaol and zingiberene. The Gingerol compound in the Ginger is said to have shown some affects on the blood vessels. When the study of use of gingerols on rat was conducted, it was proved that these gingerols stimulated blood vessel contractions in mouse and rat tissue samples by regulating signaling molecules known as eicosanoids. The reason why ginger is now considered to be an aphrodisiac food is because the same agents that lend ginger its medicinal properties are also responsible for stimulating arousal due to the warm, spicy scent and taste them impart.

Various therapies are been done with the use of ginger to treat sexual dysfunction. One of the therapies is known as Ginger Moxa. Ginger moxa is a therapy used in Chinese medicine to treat impotence that marries the properties of ginger with practice of moxibustion, a technique that involves burning selected herbs at various acupuncture points along the body. Regular intake of ginger in any form has been proved as a better and very effective option of treating erectile dysfunction. Ginger oil direct application on penis showed fast effect on erection without any side effects caused by chemical drugs.

\section{Maximum bioavailability}

The herbal composition if used as an oral formulation its bioavailability will be poor compared to a topical (external) applications on penis. To give good bioavailability, it was found that this oil with its nano particle size did give maximum bioavailability effect.

\section{Summary of the invention}

The present invention relates to a topical pharmaceutical composition for treatment of erectile dysfunction in men. The composition for treating disorder, preferably formed of fenugreek oil, watermelon oil, ginger oil and ozonated olive oil

\section{Examples of pre-clinical results}

The following results were obtained at the clinic of Dr. B. Khasawneh.

\section{Dr B.Khaswneh 3 surveys to date}
a. Age group: 26-65 for Premature Ejaculation (PE Group)
b. Number : 24 patients
c. Time applied 15 minutes before intercourse. 
d. Intercourse Time increased by 15-25 minutes.

Summary: Enhanced and long pleasure, for both men and their partners.

a. Star rating 5 .

b. Age group 50-75 for Erectile Dysfunction(ED Group).

c. Number of patients: 24 patients.

d. Time applied before intercourse: 10 minutes.

Summary: Totally fantastic, thought they were back in their twenties, it gave them hard erection for the first time in years, excellent product with complete satisfaction for both men and their women.

a. Star rating $4+$.

b. Age group 30-70 for Erectile Dysfunction and Premature Ejaculation combined (ED and PE Group).

c. Number of patients: 24 patients.

d. Time applied before intercourse: 15 minutes.

Summary: Perfect, it solved ED and PE together and changed our sex life naturally.

a. Star rating $5+$.

\section{Safety and toxicity study}

Toxicity study performed on mice in the animal house showed that the composition is free of adverse effects especially on liver, kidneys, lipid and other body organs.

\section{Conclusion}

This patented botanical nano oil mix is expected to help hundreds of millions of impotent people who suffer from ED and PE worldwide since it works on a number of mechanisms: Ginger for enhancing blood circulation; Citrulline works on vasolating blood vessels, oilive oil works on liberating Nitric Oxide, and fenugreek extract works on improving libido. Double blind is still needed to give more reliable results.

\section{Acknowledgements}

Efforts of Dr. B. Khasawneh during the course of this study is highly appreciated.

\section{Conflict of interest}

The author declares no conflict of interest.

\section{References}

1. Baker SL. Herb fenugreek could be natural Viagra, new study finds. USA: Natural News; 2011.

2. David Aranda. Olive oil sex. USA; 2014.

3. Julie Butleron. Tomatoes and Olive Oil May Reduce Impotence. USA: Olive Oil Times; 2012.

4. Mike Adams. Review of global healing center: ozonated skin salve, oxy-powder, slimirex and other revolutionary health products. USA: Natural News; 2008.

5. Watermelon vs erectile dysfunction (ED)? USA: Haltips; 2011.

6. Can Ginger Cure Erectile Dysfunction? USA: Smart Living Network; 2012. 